\title{
Methylation of Werner syndrome protein is associated with the occurrence and development of invasive meningioma via the regulation of $\mathrm{Myc}$ and $\mathrm{p53}$ expression
}

\author{
PUXIAN LI, SHUYU HAO, ZHIYONG BI, JUNTING ZHANG, ZHEN WU and XIAOHUI REN \\ Department of Neurosurgery, Beijing Tian Tan Hospital, Capital Medical University, Beijing 100050, P.R. China
}

Received December 31, 2014; Accepted May 8, 2015

DOI: $10.3892 / \mathrm{etm} .2015 .2519$

\begin{abstract}
The aim of the present study was to investigate the positive rate of Werner syndrome protein (WRN) methylation in meningioma patients, and further assess the association between WRN methylation and the occurrence of meningioma. A total of 56 consecutive meningioma patients and 26 healthy individuals were enrolled in the study. A methylation-specific polymerase chain reaction assay was performed to detect the positive rate of WRN methylation in the peripheral blood and tissue samples collected from the recruited subjects. In addition, western blot analysis was performed to determine the protein expression levels of WRN, Myc and p53 in the peripheral blood and tissue samples. The positive rate of WRN methylation in the peripheral blood of the meningioma group was increased when compared with the control group $(\mathrm{P}<0.05)$. In addition, the protein expression levels of WRN were significantly decreased in the peripheral blood and tissue samples collected from the individuals with a positive WRN methylation status $(\mathrm{P}<0.05)$, as compared with the samples without WRN methylation. Furthermore, the protein expression levels of Myc and p53 were increased in the peripheral blood and tissue samples that exhibited positive WRN methylation when compared with those without WRN methylation $(\mathrm{P}<0.05)$. Therefore, WRN methylation was demonstrated to be associated with the occurrence and development of invasive meningioma, possibly through the regulation of Myc and p53 expression.
\end{abstract}

\section{Introduction}

Meningiomas are one of the most common types of intracranial tumor, with the incidence rate of meningiomas $(\sim 19.2 \%)$ ranking second among intracranial tumors worldwide (1). At an early stage, meningiomas are benign tumors. However,

Correspondence to: Dr Junting Zhang, Department of Neurosurgery, Beijing Tian Tan Hospital, Capital Medical University, 6 Tiantan Xili, Beijing 100050, P.R. China

E-mail: zjt999@163.com

Key words: Werner syndrome protein, meningioma, methylation previous studies $(2,3)$ have demonstrated that meningiomas exhibit several metastatic malignant forms, with characteristics of fast-growth, invasion, metastasis and easy to relapse. The malignancy degree of meningioma increases with the increasing tumor grade (4).

Methylation is involved in the modification of heavy metals and the regulation of gene expression. DNA methylation in vertebrates includes global hypomethylation and regional hypermethylation, which typically occurs at cytosine-phosphate-guanine $(\mathrm{CpG})$ sites. DNA methylation is associated with the activation and expression of cancer genes (5), and the process of DNA methylation has been demonstrated to play an important role in the occurrence and development of tumors at an early stage (6-8).

Werner syndrome protein $(\mathrm{WRN})$ is a protein-coding gene that is associated with a variety of diseases, including Werner syndrome and Rothmund-Thomson syndrome (9). The WRN gene is located on the short arm of chromosome 8 between positions 12 and 11.2, and the promoter of the WRN gene has been shown to be methylated in numerous types of malignant tumor $(10,11)$. A previous study demonstrated that a polymorphism of the WRN gene was associated with the risk of developing a meningioma (12). However, the association between WRN methylation and the occurrence of meningiomas remains unclear, despite the incidence of meningiomas being associated with the abnormal expression of a variety of genes (13).

In the present study, the methylation status of the WRN promoter was detected in the peripheral blood and tissue samples of patients with meningioma using a methylation-specific polymerase chain reaction (PCR) assay. Thus, the association between WRN methylation and the incidence of meningioma was further investigated.

\section{Materials and methods}

Reagents. A DNA extraction kit and a total protein extraction kit were purchased from TIANGEN Biotech Co., Ltd. (Beijing, China). An EZ DNA Methylation-Direct ${ }^{\mathrm{TM}}$ kit was purchased from Zymo Research Corporation (Irvine, CA, USA). A rabbit anti-human WRN polyclonal antibody (ab200), a rabbit anti-human Myc polyclonal antibody (ab9106) and a mouse anti-human p53 monoclonal antibody (ab26) were purchased from Abcam (Cambridge, MA, USA). In addition, a horse- 
radish peroxidase (HRP)-conjugated goat anti-mouse IgG polyclonal secondary antibody (ab6789) and a goat anti-rabbit IgG polyclonal antibody (ab6721) were purchased from Abcam. BeyoECL Plus enhanced chemiluminescence reagent was obtained from the Beyotime Institute of Biotechnology (Suzhou, China).

Patients. A total of 56 consecutive patients with meningioma were enrolled in the study (meningioma group), of which 31 individuals were male and 25 patients were female. The age of the 56 patients ranged between 9 and 76 years, with an average age of 47.6 years. The course of the disease ranged between 2 and 51 months, with an average disease course of 21.3 months. With regard to the control group, 26 healthy individuals were enrolled in the study (control group). Peripheral blood samples were collected from the meningioma patients and the healthy individuals. In addition, meningioma tissues were collected from the meningioma patients, while healthy arachnoid tissues were collected from the healthy individuals in the control group. Prior written and informed consent was obtained from every patient, and the study was approved by the Ethics Review Board of the Capital Medical University (Beijing, China).

Detection of DNA methylation. DNA from the peripheral blood and tissue samples was extracted using the DNA extraction kit, in accordance with the manufacturer's instructions. The detection of the DNA methylation status was performed using the EZ DNA Methylation-Direct ${ }^{\mathrm{TM}}$ kit, in accordance with the manufacturer's instructions. DNA methylation was detected using a methylation-specific PCR assay, where DNA without the presence of methylation was used as a control. The primer sequences for positive WRN gene methylation were as follows: Forward, 5'-CGGGTAGGGGTATCGTTCGC-3' and reverse, 5'-CGATATCCGAAATCAAACGACG-3'. The primer sequences for negative WRN gene methylation were as follows: Forward, 5'-GTAGTTGGGTAGTAGGGGTATTGTTTGT-3' and reverse, 5'-CCAATATCCAAAATCAAACAACAAC-3'.

Western blot analysis. Total proteins from the peripheral blood and tissue samples were extracted using the total protein extraction kit. The total proteins were separated by $12 \%$ SDS-PAGE, and subsequently analyzed by immunoblotting, where $\beta$-actin was used as an internal control. Briefly, the membrane was blocked with 5\% non-fat milk at room temperature for $1 \mathrm{~h}$. The membrane was then incubated with primary antibodies at $4^{\circ} \mathrm{C}$ overnight, prior to washing. The membrane was incubated with secondary antibodies at room temperature for $1 \mathrm{~h}$, The primary antibodies used included a rabbit anti-human WRN polyclonal antibody (1:2,000), a rabbit anti-human Myc polyclonal antibody (1:500) and a mouse anti-human p53 monoclonal antibody $(1: 1,000)$. The secondary antibodies used in the experiment were a HRP-conjugated goat anti-rabbit IgG polyclonal antibody $(1: 3,000)$ and a HRP-conjugated goat anti-mouse IgG polyclonal antibody $(1: 5,000)$. The blots were detected using BeyoECL Plus enhanced chemiluminescence reagent, and image quantifications were performed using ImageLab software, version 4.1 (Bio-Rad Laboratories, Inc., Hercules, CA, USA). The experiments were repeated a minimum of three times.
Statistical analysis. All results are expressed as the mean \pm standard deviation. The statistical analyses were performed using SPSS software, version 17.0 for Windows (SPSS, Inc., Chicago, IL, USA). The least significant difference t-test was used to analyze the comparisons between groups and for the analysis of paired data, where $\mathrm{P}<0.05$ was considered to indicate a statistically significant difference.

\section{Results}

Positive rate of WRN methylation is increased in the peripheral blood and tissues of meningioma patients. To investigate the levels of WRN methylation in the peripheral blood and tissues, a methylation-specific PCR assay was performed. As shown in Table I, the positive rate of WRN methylation was 55.36 and $0.00 \%$ in the peripheral blood of the meningioma and control groups, respectively. Furthermore, the positive rate of WRN methylation was 60.71 and $7.69 \%$ in the tissues of the meningioma and control groups, respectively. Thus, the positive rate of WRN methylation in the peripheral blood of the meningioma group was significantly increased when compared with the control group $(\mathrm{P}<0.05$; Fig. 1A). In addition, the positive rate of WRN methylation in the tissues of the meningioma group was significantly increased when compared with the control group $(\mathrm{P}<0.05$; Fig. 1B). These results indicated that the positive rate of WRN methylation was increased in the peripheral blood and tissues of meningioma patients.

Protein expression of WRN is inhibited following WRN methylation in the peripheral blood and tissues. To determine the protein expression levels of WRN in the peripheral blood and tissue samples, western blot analysis was performed. As shown in Fig. 2A, the protein expression levels of WRN in the peripheral blood samples with positive WRN methylation were decreased when compared with those without WRN methylation $(\mathrm{P}<0.05)$. In addition, the protein expression levels of WRN in the tissues with WRN methylation were decreased when compared with those without WRN methylation $(\mathrm{P}<0.05)$. Therefore, in the peripheral blood and tissue samples with positive WRN methylation, WRN protein expression levels were significantly decreased. These results indicated that the protein expression of WRN is inhibited following WRN methylation in the peripheral blood and tissues.

Myc and p53 protein expression levels are increased in the peripheral blood and tissues with WRN methylation. To investigate the effect of WRN methylation on the regulation of tumor gene expression, western blot analysis was performed to detect the protein expression levels of Myc and p53. Myc and p53 are regulated by WRN in the process of tumor development (14). As shown in Fig. 3A, the expression levels of Myc in the peripheral blood of the patients with positive WRN methylation were increased when compared with those without WRN methylation $(\mathrm{P}<0.05)$. In addition, the expression levels of Myc in the tissue samples with WRN methylation were increased when compared with those without WRN methylation $(\mathrm{P}<0.05)$ (Fig. 3B). As shown in Fig. 4A, the expression levels of p53 in the peripheral blood samples with positive WRN methylation were increased when compared with those without WRN methylation $(\mathrm{P}<0.05)$. Furthermore, the expression levels of p53 in the tissue samples 
Table I. Positive rate of WRN methylation in the peripheral blood and tissues.

\begin{tabular}{|c|c|c|c|c|c|c|c|c|c|}
\hline \multirow[b]{2}{*}{ Groups } & \multirow[b]{2}{*}{ Cases (n) } & \multicolumn{4}{|c|}{ Methylation in the peripheral blood } & \multicolumn{4}{|c|}{ Methylation in the tissues } \\
\hline & & Yes (n) & No (n) & Positive rate (\%) & P-value & Yes (n) & No (n) & Positive rate (\%) & P-value \\
\hline Control & 26 & 0 & 26 & 0 & & 2 & 24 & 7.69 & \\
\hline Meningioma & 56 & 31 & 25 & 55.36 & $<0.001$ & 32 & 24 & 60.71 & $<0.001$ \\
\hline
\end{tabular}

WRN, Werner syndrome protein.

A

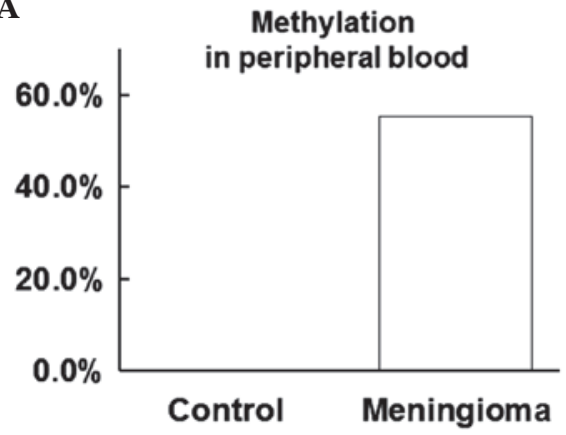

B

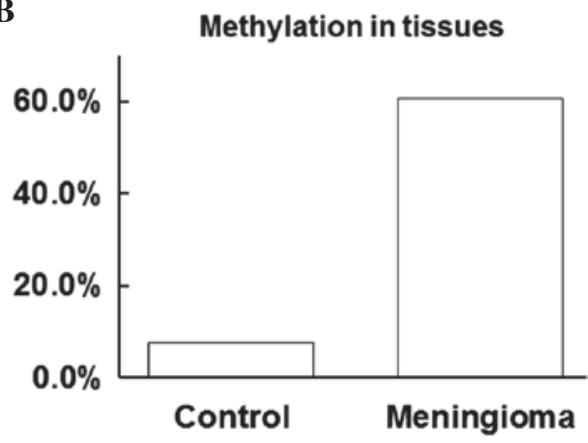

Figure 1. Positive rate of Werner syndrome protein (WRN) methylation in the peripheral blood and tissues. A methylation-specific polymerase chain reaction assay was performed to detect the positive rate of WRN methylation in the (A) peripheral blood and (B) tissue samples collected from the meningioma patients and the healthy control group.

A

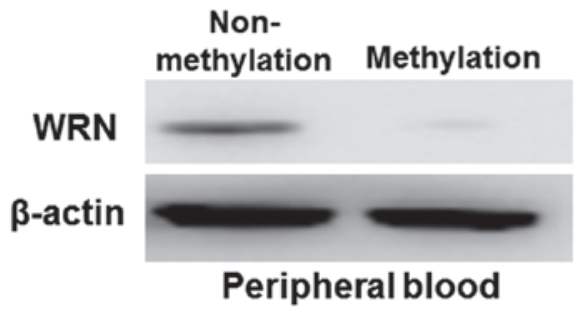

B

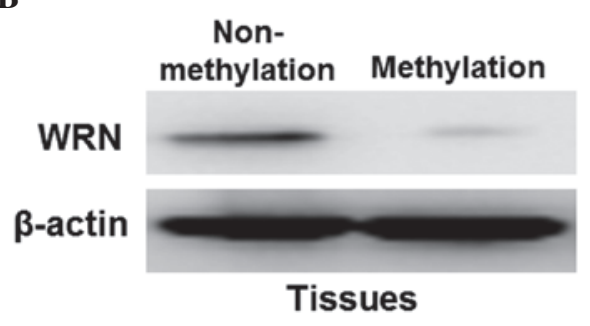

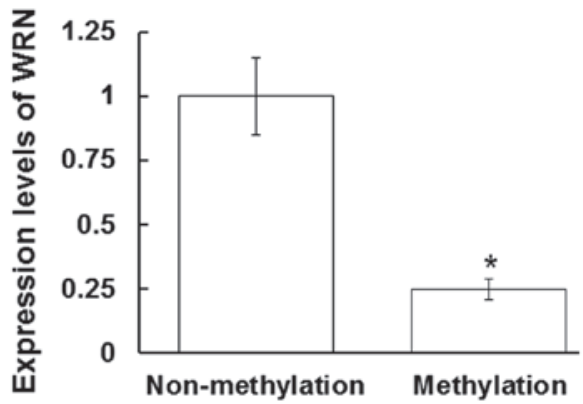

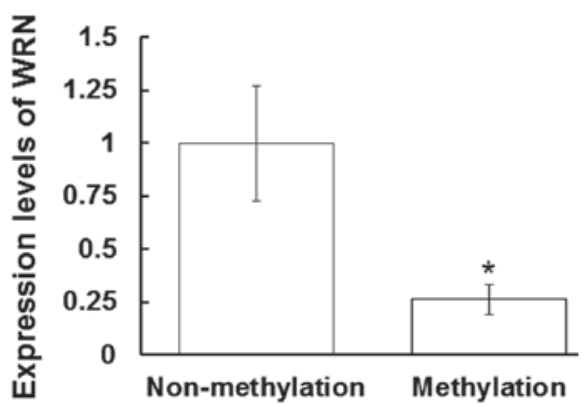

Figure 2. Protein expression levels of WRN in the peripheral blood and tissues. Total proteins from the peripheral blood and tissue samples were harvested, separated by $12 \%$ SDS-PAGE and analyzed by western blot analysis, where $\beta$-actin was used as a loading control. Protein expression levels of WRN in the (A) peripheral blood and (B) tissues were detected using a rabbit anti-human WRN polyclonal antibody $(1: 2,000)$. Left panel, representative western blot; right panel, quantitative assessment of the western blot analysis results. " $\mathrm{P}<0.05$, vs. non-methylation group. WRN, Werner syndrome protein.

with positive WRN methylation were increased when compared with those without WRN methylation (P<0.05; Fig. 4B). These results indicated that the protein expression levels of Myc and p53 were increased in the peripheral blood and tissue samples that exhibited positive WRN methylation.

\section{Discussion}

The incidence of meningiomas has increased in recent years (15). The occurrence of tumors is associated with the activation of cancer genes and the inactivation of tumor 
A

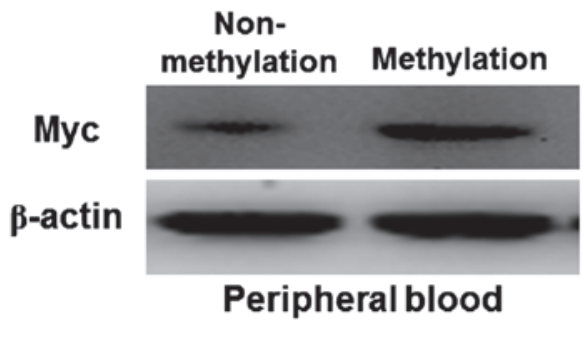

B

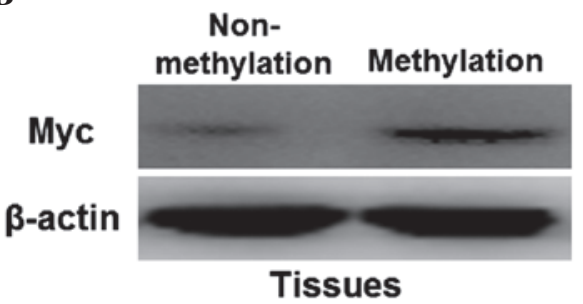

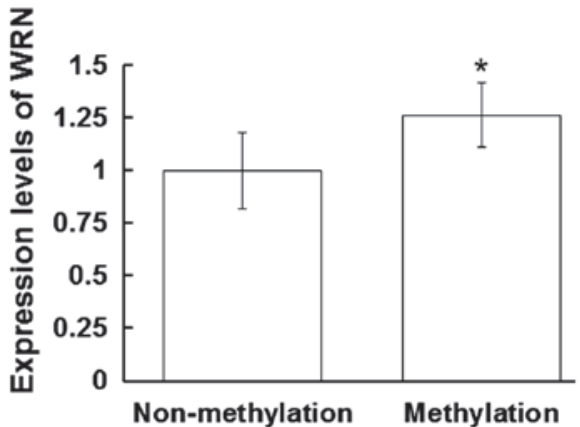

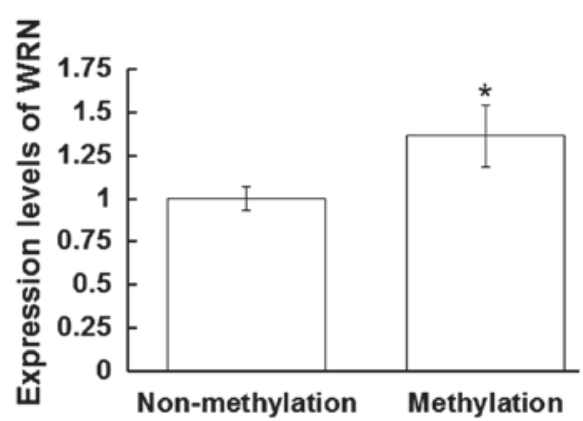

Figure 3. Expression levels of Myc in the peripheral blood and tissues. Total proteins from the peripheral blood and tissue samples were harvested, separated by $12 \%$ SDS-PAGE and analyzed with western blot analysis, where $\beta$-actin was used as a loading control. Protein expression levels of Myc in the (A) peripheral blood and (B) tissues were detected using a rabbit anti-human Myc polyclonal antibody (1:500). Left panel, representative western blot; right panel, quantitative assessment of the western blot analysis results. ${ }^{*} \mathrm{P}<0.05$, vs. non-methylation group. WRN, Werner syndrome protein.

A

p53

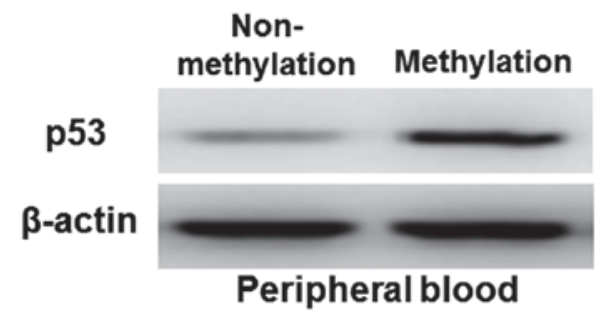

B p53

$\beta$-actin

Nonmethylation Methylation

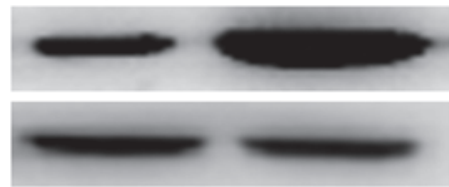

Tissues
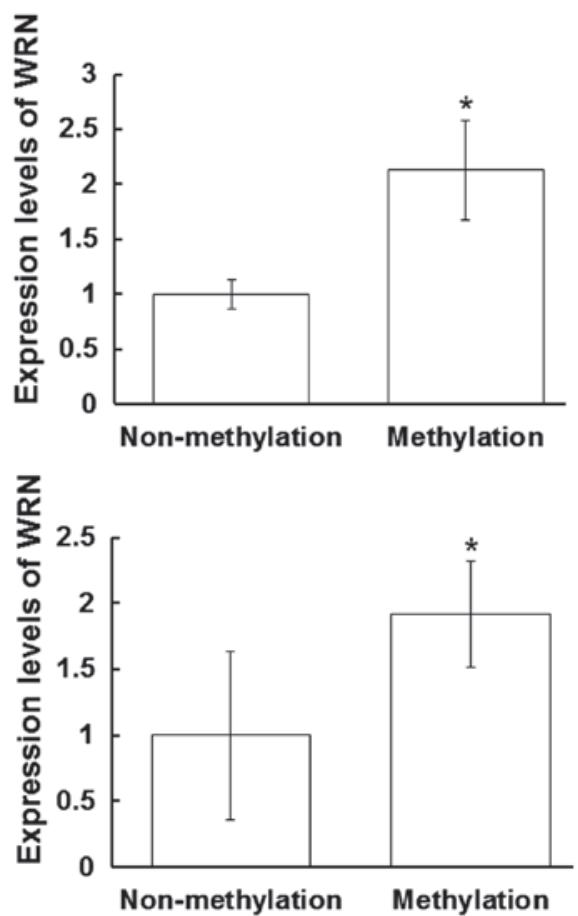

Figure 4. Expression levels of $\mathrm{p} 53$ in the peripheral blood and tissues. Total proteins from the peripheral blood and tissue samples were harvested, separated by $12 \%$ SDS-PAGE and analyzed by western blot analysis, where $\beta$-actin was used as a loading control. Protein expression levels of p53 in the (A) peripheral blood and (B) tissues were detected using a mouse anti-human p53 monoclonal antibody $(1: 1,000)$. Left panel, representative western blot; right panel, quantitative assessment of the western blot analysis results. ${ }^{*} \mathrm{P}<0.05$, vs. non-methylation group. WRN, Werner syndrome protein.

suppressor genes $(16,17)$. Tumor suppressor genes exist in normal cells of every healthy individual. When the tumor suppressor gene is activated, cell proliferation is inhibited. However, when the tumor suppressor gene is suppressed, the role of the tumor suppressor is eradicated (18).
In the human genome, fragments that are rich in $\mathrm{CpG}$ dinucleotides are known as $\mathrm{CpG}$ islands, and these are primarily located in the promoter region and the first exon region of the gene (17). Approximately $60 \%$ of the promoter region is estimated to contain CpG islands (19). The occurrence and 
development of tumors are associated with DNA methylation. A number of tumor-associated tumor suppressor genes are methylated in the $\mathrm{CpG}$ islands of the gene promoter, which has been shown to affect the conformation and stability of DNA, and ultimately regulate gene expression (20). WRN plays an important role in DNA damage repair processes, which are involved in the maintenance of gene stability $(14,21)$. High expression levels of WRN have been shown to inhibit the regulatory function of Myc in the process of cell aging (14).

In the present study, the results demonstrated that the positive rate of WRN methylation in the peripheral blood of the meningioma group was increased when compared with the control group. In addition, the protein expression levels of WRN were significantly decreased in the peripheral blood and tissue samples of the individuals exhibiting positive WRN methylation, indicating that the expression levels of WRN may be inhibited by the regulation of WRN methylation. Furthermore, the protein expression levels of Myc and p53 were increased in the peripheral blood and tissue samples from the patients with positive WRN methylation, as compared with those that did not exhibit WRN methylation. Therefore, these results indicate that WRN methylation may be associated with tumorigenesis.

In conclusion, the methylation of WRN was demonstrated to be associated with the occurrence and development of invasive meningioma, possibly through the regulation of Myc and p53 protein expression. Subsequently, the diagnosis of meningioma may be predicted by the detection of WRN methylation. Therefore, the detection of the WRN methylation status may play an important role in the diagnosis and treatment of meningioma patients.

\section{Acknowledgements}

The study was supported by grants from the Natural Science Foundation of China (no. 81341059) and the Beijing Nova Program (no. 2012033). The authors thank Professor Hong Wan from the Beijing Neurosurgery Institute of the Capital Medical University for the support provided during the study.

\section{References}

1. Chamberlain MC, Glantz MJ and Fadul CE: Recurrent meningioma: Salvage therapy with long-acting somatostatin analogue. Neurology 69: 969-973, 2007.

2. Park KJ, Yu MO, Song NH, et al: Expression of astrocyte elevated gene-1 (AEG-1) in human meningiomas and its role in cell proliferation and survival. J Neurooncol 121: 31-39, 2015.
3. Erkutlu I, Buyukhatipoglu H, Alptekin M, et al: Spinal drop metastases from a papillary meningioma: A case report and review of the literature: Utility of CSF sampling. Med Oncol 26: 242-246, 2009

4. Louis DN, Ohgaki H and Wiestler OD: The 2007 WHO classification of tumours of the central nervous system. Acta Neuropathol 114: 97-109, 2007.

5. Hatziapostolou M and Iliopoulos D: Epigenetic aberrations during oncogenesis. Cell Mol Life Sci 68: 1681-1702, 2011.

6. Alvarez H, Opalinska J, Zhou L, et al: Widespread hypomethylation occurs early and synergizes with gene amplification during esophageal carcino-genesis. PLoS Genet 7: e1001356, 2011.

7. Simmer F, Brinkman AB, Assenov Y, et al: Comparative genome-wide DNA methylation analysis of colorectal tumor and matched normal tissues. Epigenetics 7: 1355-1367, 2012.

8. Jordà M and Peinado MA: Methods for DNA methylation analysis and applications in colon cancer. Mutat Res 693: 84-93, 2010.

9. Furuichi Y: Premature aging and predisposition to cancers caused by mutations in RecQ family helicases. Ann NY Acad Sci 928: 121-131, 2001.

10. Wang L, Xie L, Wang J, et al: Correlation between the methylation of SULF2 and WRN promoter and the irinotecan chemosensitivity in gastric cancer. BMC Gastroenterol 13: 173, 2013.

11. Kawasaki T, Ohnishi M, Suemoto Y, et al: WRN promoter methylation possibly connects mucinous differentiation, microsatellite instability and $\mathrm{CpG}$ island methylator phenotype in colorectal cancer. Mod Pathol 21: 150-158, 2008.

12. Wang K, Hao SY, Huang GY, et al: Association study between polymorphism of WRN gene and meningioma in Chinese population. Zhong Guo Wei Qin Xi Shen Jing Wai Ke Za Zhi 17: 248-251, 2012 (In Chinese).

13. He S, Pham MH, Pease M, et al: A review of epigenetic and gene expression alterations associated with intracranial meningiomas. Neurosurg Focus 35: E5, 2013.

14. Opresko PL, Calvo JP and von Kobbe C: Roles for Werner syndrome protein in the promotion of tumor cell growth. Mech Ageing Dev 128: 423-436, 2007.

15. Campbell BA, Jhamb A, Maguire JA, et al: Meningiomas in 2009: Controversies and future challenges. Am J Clin Oncol 32: 73-85, 2009.

16. Vranic A, Peyre M and Kalamarides M: New insights into meningioma: From genetics to trials. Curr Opin Oncol 24: 660-665, 2012.

17. Curtin K, Slattery ML and Samowitz WS: CpG island methylation in colorectal cancer: Past, present and future. Patholog Res Int 2011: 902674, 2011.

18. Fearon ER: Molecular genetics of colorectal cancer. Annu Rev Pathol 6: 479-507, 2011.

19. Feltus FA, Lee EK, Costello JF, et al: Predicting aberrant CpG island methylation. Proc Natl Acad Sci USA 100: 12253-12258, 2003.

20. Takai D and Jones PA: Comprehensive analysis of CpG islands in human chromosomes 21 and 22. Proc Natl Acad Sci USA 99: 3740-3745, 2002.

21. Bacolla A, Wang G, Jain A, et al: Non-B DNA-forming sequences and WRN deficiency independently increase the frequency of base substitution in human cells. J Biol Chem 286: 10017-10026, 2011. 